\title{
Exploring the structure of hadronic showers and the hadronic energy reconstruction with highly granular calorimeters
}

\author{
Vladimir Bocharnikov ${ }^{1, *}$ \\ Deutsches Elektronen-Synchrotron DESY, \\ Notkestr. 85, 22607 Hamburg, Germany \\ E-mail: vladimir.bocharnikov@desy.de
}

Prototypes of electromagnetic and hadronic imaging calorimeters developed and operated by the CALICE collaboration provide an unprecedented wealth of highly granular data of hadronic showers with a variety of active sensor elements and different absorber materials. In this article, we discuss detailed measurements of the spatial and the time structure of hadronic showers to characterise the different stages of hadronic cascades in the calorimeters and perform particle identification techniques. The high granularity of the detectors is exploited in the reconstruction of hadronic energy, both in individual detectors and combined electromagnetic and hadronic systems, making use of software compensation and semi-digital energy reconstruction algorithms. The results on hadronic shower measurements are obtained using different electromagnetic and hadronic calorimeters, with silicon, scintillator and gaseous active elements, and confronted with GEANT4-based simulations using different hadronic physics models.

\footnotetext{
*** The European Physical Society Conference on High Energy Physics (EPS-HEP2021), ***

*** 26-30 July $2021 * * *$

*** Online conference, jointly organized by Universität Hamburg and the research center DESY ***
}

${ }^{1}$ on behalf of the CALICE collaboration

${ }^{*}$ Speaker 


\section{Introduction}

One of the key components for the success of high-energy physics experiments is detector performance. The physics program for future $e^{+} e^{-}$collider experiments sets the goal to reconstruct jet energies with a resolution of 3-4\% in the energy range between $\sim 50 \mathrm{GeV}$ and several hundreds $\mathrm{GeV}$. The most promising solution to achieve this goal is the so called Particle Flow Approach (PFA). PFA aims to reconstruct each individual particle within a hadronic jet and measure its energy with the best suited detector subsystem. To ensure the correct assigment of tracks and calorimeter information for charged particles, high granularity of the calorimeter system is required.

The CALICE collaboration develops such highly granular calorimeters. There are several sampling calorimeter designs with different absorber materials and readout systems: electromagnetic calorimeters with tungsten absorber and analog readout using silicon (Si-W ECAL) or scintilatorSiPM (ScECAL) active elements, hadronic calorimeters with either stainless steel or tungsten absorbers and scintilator-SiPM with analog readout (AHCAL) or resistive plate chambers with digital/semi-digital readout (DHCAL/SDHCAL).

The imaging capabilities of highly granular calorimeters can be used for GEANT4 modeling validation, testing different PFA algorithms (such as ref. [1]) and improving hadronic energy resolution by use of additional spatial information. This article gives an overview on hadronic shower measurement studies using highly granular calorimeter prototypes by the CALICE collaboration with selection of recent results.

\section{Topological shower analyses}

The fine transverse and longitudinal segmentation of CALICE prototypes provides an opportunity to study in detail the spatial development of hadronic showers. Both transverse and longitudinal energy density distributions of hadronic showers can be studied and compared with simulations.

The longitudinal development of hadronic showers is important for the estimation of leakage effects while radial energy density distributions give understanding of limits for PFA algorithms in disentangling signals from overlapping showers. Examples of radial profiles for DHCAL and Si-W ECAL are shown in Fig.1. Modeling shows reasonable performance, with deviation up to $30 \%$ at large radii and visible dependence on the GEANT4 version. More information can be found in refs. [2-5].

To study the inner structure of hadronic showers with highly granular calorimeters, it is possible to reconstruct track segments corresponding to secondary minimum ionising particles [6-8].This allows an indirect comparison of shower composition between data and simulations. As an example the mean track multiplicity as a function of the charged pion beam energy is shown in Fig. 2. Simulations tend to underestimate the track multiplicity, but in general the agreement stays within $10 \%$. In addition to hadronic shower structure studies, reconstructed tracks can be used for in-situ calibration of the calorimeter, which is important for operation of a full scale collider detector.

Highly granular calorimeters allow to classify events by the incoming particle type using the topology of the event image. The main motivation for such a classification in a beam test environment is the rejection of beam contamination before the determination of such parameters as the intrinsic hadronic energy resolution. In addition, calorimeter based particle identification is 

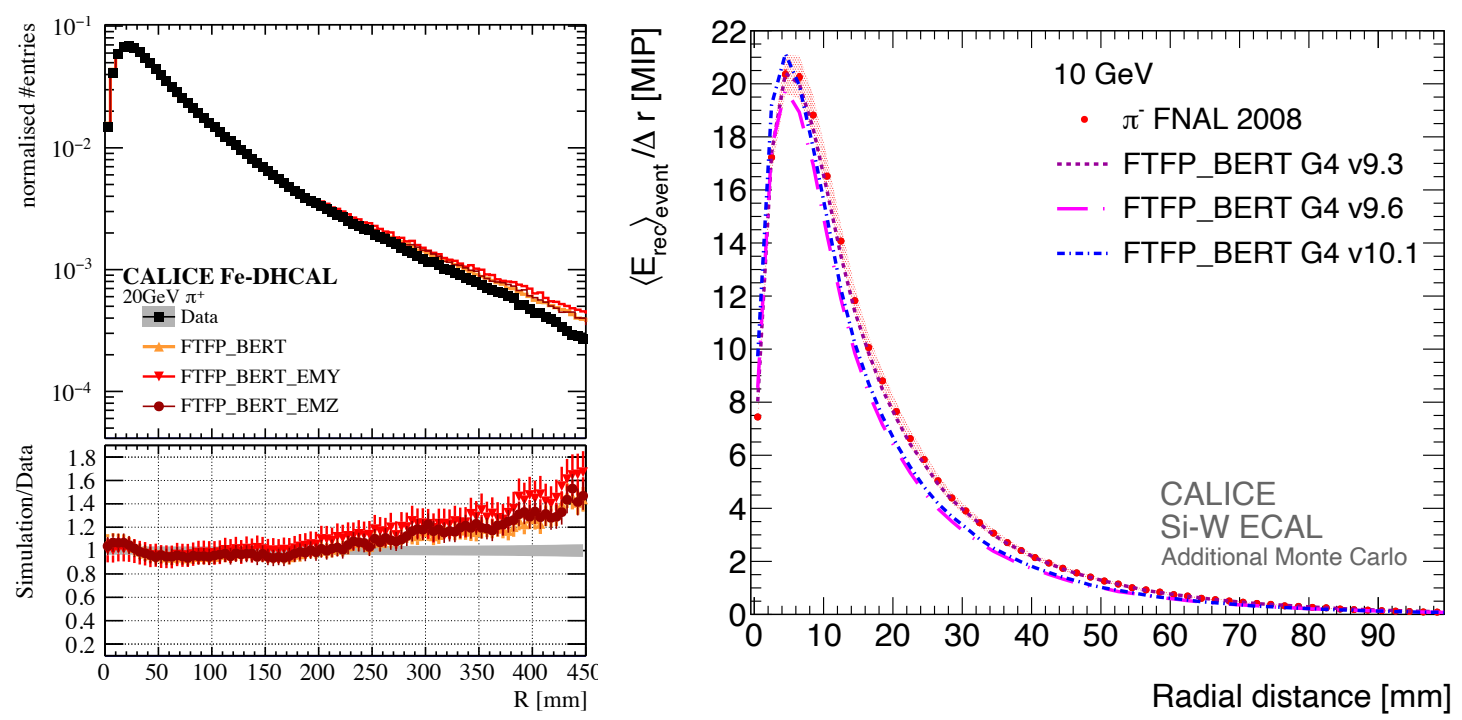

Figure 1: Left: radial profile example for $20 \mathrm{GeV} \pi^{+}$run measured in DHCAL with comparison to simulations using several physics lists [3]. Right: radial profile example for $10 \mathrm{GeV} \pi^{-}$measured by Si-W ECAL with comparison to simulations using different versions of GEANT4 [5]
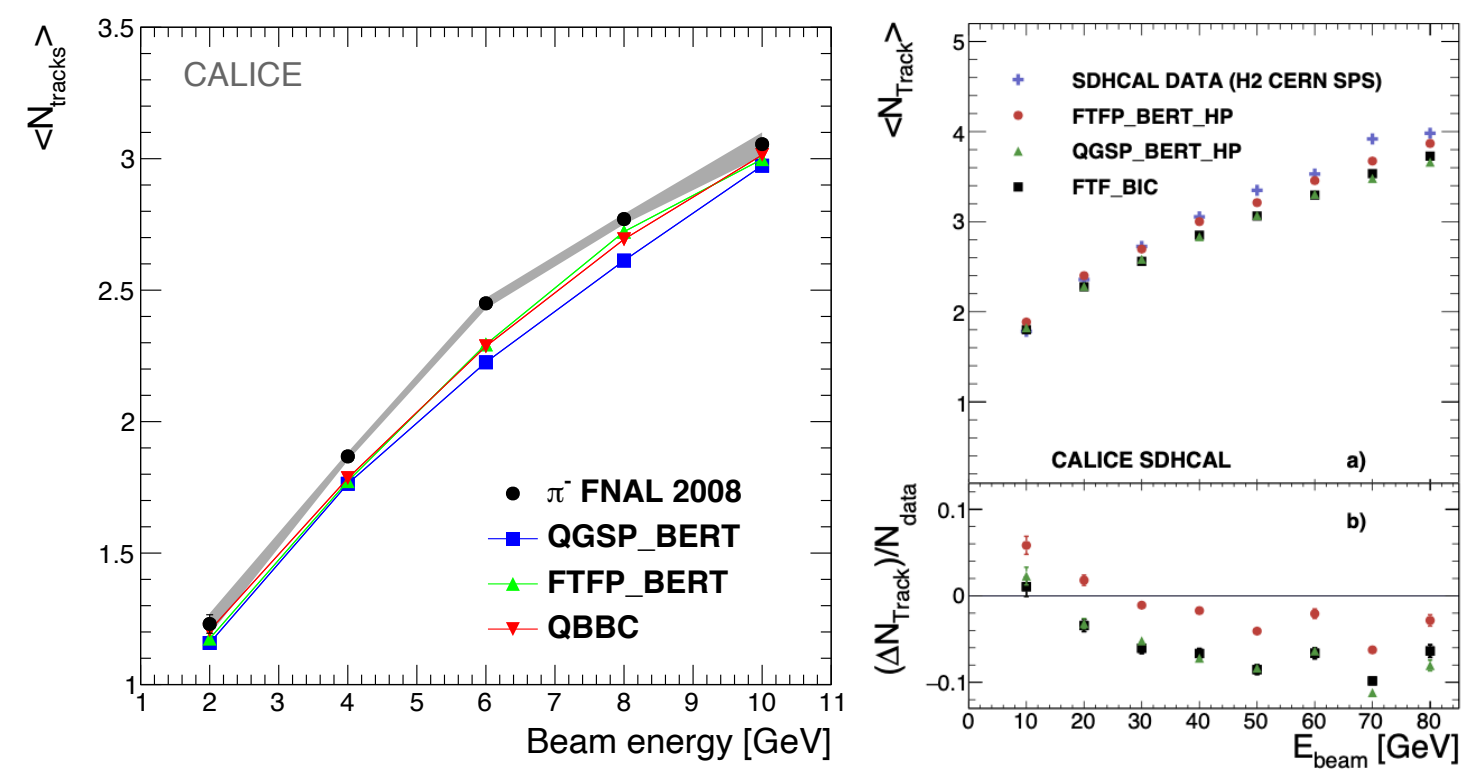

Figure 2: Energy dependence of mean track multiplicity within hadronic showers with comparison to simulations measured by Si-W ECAL [7] (left), SDHCAL[8] (right). 

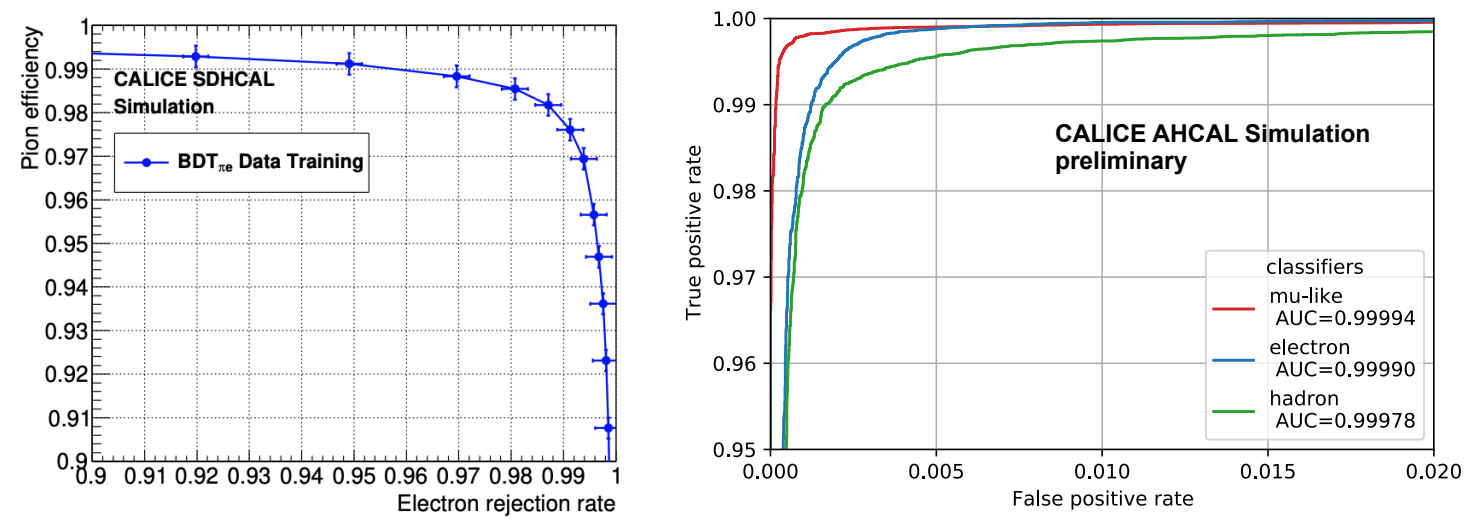

Figure 3: Left: SDHCAL particle identification performance. Pion efficiency versus electron rejection rate in energy range from $10 \mathrm{GeV}$ to $80 \mathrm{GeV}$. Right: AHCAL particle identification performance. ROC-curves for 3 output classifiers (muon, electron and hadron) with corresponding Area Under the Curve (AUC) metric applying BDT model to balanced test dataset composed from simulated muons, electroms and showering charged pions in energy range from $10 \mathrm{GeV}$ to $200 \mathrm{GeV}$.

essential for physics analysis and performance of PFA algorithms in a collider detector scenario. Discriminating variables based on event topologies of showering charged pions, electrons and muons obtained from simulations and test beam data are used to train Boosted Decision Tree (BDT) classification models [9]. The method shows excellent signal efficiency and background rejection rate which is demonstrated with an example of pion signal efficiency versus electron rejection rate shown on the left part of Fig. 3. Usage of BDT method leads to more signal statistics than standard cut-based method ([10]).

A similar analysis with the AHCAL technological prototype is in progress. Three classifiers corresponding to particle types are trained simultaneusly using 11 topological variables of simulated events in energy range from 10 to $200 \mathrm{GeV}$. The sum of the three classifiers is normalised to unity, so the BDT model output can be interpreted as probability of an event to belong to the coresponding class. Preliminary results on a simulated test dataset show excellent separation performance. The right part of Fig. 3 shows Receiver-Operator Characteristic (ROC) curves for muon, electron and hadron classifiers applied to the test dataset obtained from simulations.

\section{Energy reconstruction}

The complex structure of hadron-induced showers with large event-by-event fluctuations of electromagnetic and hadronic components limits the energy resolution of calorimeters. In addition to the total energy deposition, highly granular calorimeters provide information about the energy density distribution and the spatial shower structure. In case of analog readout this information can be used for software compensation (SC) methods which aim to correct the reconstructed for the difference in detector response to hadronic and electromagnetic components of hadron-induced showers. An example of applying SC to the pion shower measurements performed by a combined highly granular scintillator calorimeter system is shown in Fig. 4. The use of the SC technique 


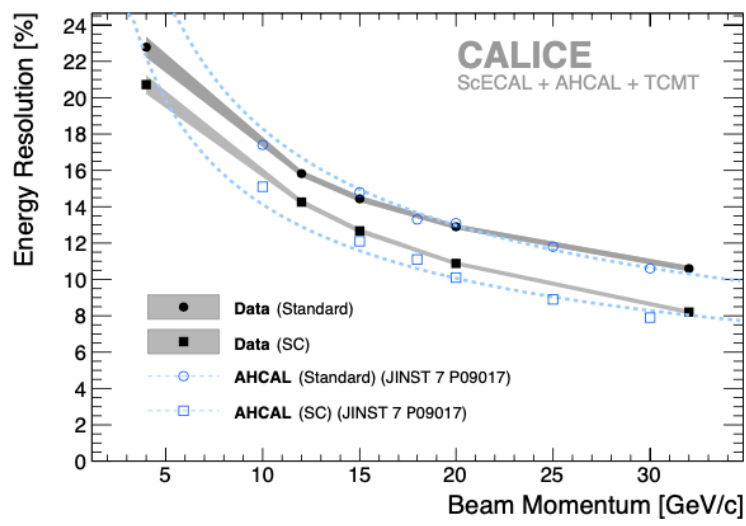

Figure 4: Single pion energy resolutions with standard and software compensation reconstruction from the combined ScECAL+AHCAL+tailcatcher system compared to resolutions obtained from AHCAL+tailcatcher [11]

significantly improves the intrinsic hadronic energy resolution of the AHCAL standalone as well as combined calorimeter system with added ScECAL in front. Similarly, multi-threshold hadronic energy reconstruction for the SDHCAL gives a significant resolution improvement over binary mode for energies higher than $30 \mathrm{GeV}$ [10].

\section{Time development of hadronic showers}

The time structure of hadronic showers is characterised by two main components: the prompt component caused by secondary electromagnetic showers and ionising particles, and the delayed energy deposition related mostly to neutron-induced processes that extend to time scales up to the order of $\mu s$. The fraction of the late component is influenced by absorber and active material properties. A dedicated study to measure and compare the time structure of hadronic showers with tungsten and steel absorbers was carried out by placing a timing layer with sub- $n s$ resolution behind W-AHCAL/Fe-SDHCAL [12]. Results of the study show that the late component is visible on the $n s$ timescale with a significant increase for tungsten compared to steel. The relative importance of late energy depositions increases with distance from the shower axis. Therefore, $1 \mathrm{~ns}$ resolution is expected to be enough to potentially improve the hadronic energy reconstruction and overlapping shower separation performance.

The second generation of CALICE prototypes, the so-called technological prototypes, are equipped with timing readout for all individual channels. Preliminary results of the AHCAL technological prototype with muon beam show that the design goal of $1 \mathrm{~ns}$ time resolution has been reached. Timing information will be exploited in upcoming results of hadronic shower measurements with CALICE prototypes.

\section{Conclusion}

This article summarises a selection of the CALICE collaboration results on hadronic shower analysis. Imaging capabilities of the CALICE highly granular calorimeter prototypes give a unique 
opportunity to explore the structure of hadronic showers and perform detailed comparison of observed test beam data with simulations. Pattern recognition and multivariate analysis techniques are performed to exploit the obtained spatial information of calorimetric images. Hadronic energy reconstruction also benefits from high granularity by use of software compensation and multithreshold reconstruction techniques. Timing readout is a promising addition to the highly granular calorimeter design. Scalable prototypes are being developed and tested with more beam test results expected in the future.

\section{References}

[1] CALICE collaboration, Tests of a particle flow algorithm with CALICE test beam data, JINST 6 (2011) P07005.

[2] CALICE collaboration, Testing hadronic interaction models using a highly granular silicon-tungsten calorimeter, Nucl. Instrum. Meth. A 794 (2015) 240 [1411. 7215].

[3] CALICE collaboration, Analysis of testbeam data of the highly granular RPC-steel CALICE digital hadron calorimeter and validation of Geant4 Monte Carlo models, NIM-A 939 (2019) 89.

[4] CALICE collaboration, Resistive Plate Chamber digitization in a hadronic shower environment, JINST 11 (2016) P06014.

[5] CALICE collaboration, Testing hadronic interaction models using a highly granular silicon-tungsten calorimeter, NIM-A 794 (2015) 240.

[6] CALICE collaboration, Track segments in hadronic showers in a highly granular scintillator-steel hadron calorimeter, JINST 8 (2013) P09001.

[7] CALICE collaboration, Characterisation of different stages of hadronic showers using the CALICE Si-W ECAL physics prototype, NIM-A 937 (2019) 41.

[8] CALICE collaboration, Tracking within hadronic showers in the CALICE SDHCAL prototype using a Hough transform technique, JINST 12 (2017) P05009.

[9] CALICE collaboration, Particle identification using Boosted Decision Trees in the Semi-Digital Hadronic Calorimeter prototype, JINST 15 (2020) P10009-P10009.

[10] CALICE collaboration, First results of the CALICE SDHCAL technological prototype, JINST 11 (2016) P04001-P04001.

[11] CALICE collaboration, Hadronic energy resolution of a combined high granularity scintillator calorimeter system, JINST 13 (2018) P12022.

[12] CALICE collaboration, The time structure of hadronic showers in highly granular calorimeters with tungsten and steel absorbers, JINST 9 (2014) P07022. 